\title{
A Robust GPS Receiver Self Survey Algorithm
}

\author{
Keunjoo Park* and John L. Crassidis ${ }^{\dagger}$
}

\begin{abstract}
To use a GPS receiver as an attitude sensor several system parameters, such as integer ambiguities, line biases and baselines, need to be first determined. This operation is called the self survey. It also may require several subsystem aspects, such as cycle slip detection and repair. In this paper, a new self survey scheme is presented that is robust to initial guess errors in the survey parameters. This new approach first uses an attitude-independent method with double-differenced phase measurements to resolve the integer ambiguities, and then determines the remaining parameters using nonlinear least-square methods. The algorithm is verified with both simulated and real GPS receiver data.
\end{abstract}

\section{Introduction}

The Global Positioning System (GPS) was originally developed for the purpose of navigation. Using a GPS receiver with pseudorange measurements, instantaneous positions as well as precise time can be determined [1]. In addition, a GPS receiver has the ability to provide attitude information by employing multiple antenna sets to measure the signal carrier phase differences [2-5]. However, when using the phase measurements the solution becomes more complicated than position determination since the phase measurements contain integer ambiguities [6-8]. Furthermore, to utilize a GPS receiver as an attitude sensor several system parameters, such as baselines, line biases and integer ambiguities, need to be first determined $[5,9,10]$. This operation to estimate all the required parameters is called the self survey.

\footnotetext{
*Senior Researcher, Dept. of Communication Satellite Systems, COMS Program Office, Korea Aerospace Research Institute, Daejeon 305-333, Republic of Korea. Tel. +82-42-860-2776, E-mail: kjp@kari.re.kr.

$\dagger$ Associate Professor, Dept. of Mechanical \& Aerospace Engineering, University at Buffalo, State University of New York, Amherst, NY 14260-4400. Tel: (716) 645-2593, ext. 2262, Fax: (716) 645-3668, E-mail: johnc@eng.buffalo.edu.
} 
In general, a self survey requires 6 to 8 hours of data to estimate baselines, line biases, integer ambiguities and the static attitude, because the sightlines, i.e., the line-of-sight (LOS) vectors between the GPS satellites and the receiver, are moving slowly relative to the motionless receiver [2]. The orbital period of the GPS satellites is approximately 12 sidereal hours. Since the GPS satellites are moving with respect to the receiver, the connections between various GPS satellites and the receiver will be on and off repeatedly. When a new GPS satellite signal is available, the integer ambiguities must be resolved first. Also, the GPS signals are often blocked for possibly several minutes [1]. This causes cycle slips or jumps in the phase measurements, because the receiver accumulates the cycles of the carrier phase. For the correct estimation of the survey parameters, cycle slip free measurements should be obtained. Altmayer enhanced the integrity of an integrated GPS/INS system by cycle slip detection and correction [11]. Since the phases change slowly, cycle slips can be successfully detected and repaired by using a polynomial fitting method [5,10]. Hence, several subsystem aspects, such as an integer ambiguity resolution routine to determine the integer number wavelengths in the phase measurements as well as a cycle slip detection and repair algorithm, are needed. Once a self survey is accomplished, the attitude determination problem can then be solved.

In this paper, a new self survey algorithm that estimates the self survey parameters is developed using linear and nonlinear estimation methods. To ensure robust convergence properties, the Levenberg-Marquardt algorithm is applied for the nonlinear estimation process. The convergence behavior of the new algorithm is investigated by Monte-Carlo like simulations. Then, simulated as well as actual GPS data collected by a commercial receiver are used to verify the new algorithm.

\section{Problem Statement}

The model of the single differenced GPS signal carrier phase between the Master Antenna (MA) and a Slave Antenna (SA) is shown in Figure 1 [12]. The $j$-th sightline vector, $\boldsymbol{s}_{j} \in \mathrm{R}^{3}$, is the unit LOS vector from the receiver to the $j$-th GPS satellite in the Earth-CenteredEarth-Fixed (ECEF) reference frame [13]. The $i$-th baseline vector, $\mathbf{b}_{i} \in \mathrm{R}^{3}$, is the relative position vector from the phase center of the MA to that of the $i$-th $\mathrm{SA}$, which is represented by cycles in the body frame. Since the wavelength of the L1 frequency of the GPS signal carrier is $\lambda=19.03 \mathrm{~cm}$, an integer (or cycle) ambiguity, $n_{i j}$, may occur either if the baseline 


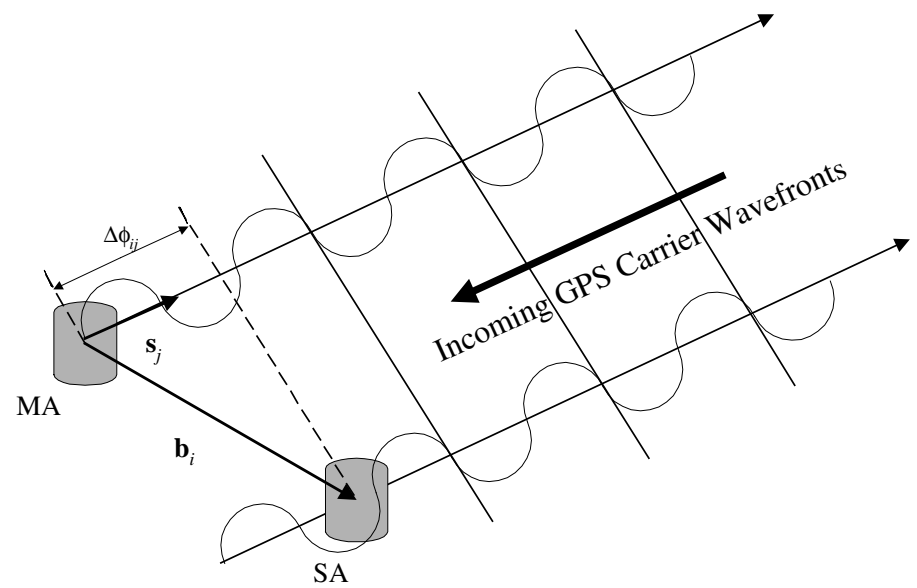

Figure 1: Planar Phase Measurement Model

is longer than the signal wavelength or when cycle slips occur due to signal loss. Since the distance between the receiver and GPS satellites is usually very large relative to the baseline length, the wavefronts of GPS signal carrier can be considered to be essentially planar [2]. Therefore, the single differenced carrier phase between the $i$-th baseline and $j$-th sightline, $\Delta \phi_{i j}$, shown in Figure 1 can be written by

$$
\Delta \phi_{i j}=\mathbf{b}_{i}^{\top} A \boldsymbol{s}_{j}+n_{i j}+\tau_{i}
$$

where $A \in \mathrm{R}^{3 \times 3}$ is the attitude matrix from the ECEF reference frame to the body frame, and $\tau_{i}$ is the line bias of the $i$-th baseline. The self survey determines the assumed constant attitude $A$, baseline in the body frame $\mathbf{b}_{i}$, integer ambiguity $n_{i j}$, and line bias $\tau_{i}$ by using the phase difference measurements that contain additive noise, denoted by $\Delta \widetilde{\phi}_{i j}$, and the sightline $\boldsymbol{s}_{j}$ information. The measurement noise is assumed to be represented by a zeromean Gaussian white-noise process with standard deviation given by $0.5 \mathrm{~cm} / \lambda=0.026$ wavelengths [2]. The sightline information is obtained from the navigation data collected by the receiver.

\subsection{Self Survey Approaches}

As can be seen in Eq. (1), the self survey estimation process is a nonlinear problem. Most self survey algorithms rely on batch-type processes for the the estimation of the assumed constant parameters [5]. Either nonlinear least squares (NLS) or a gradient search method can directly be used, however, the attitude parameters and the baselines are not indepen- 
dent [10]. Therefore, a singularity in the derived Hessian matrix occurs. Alternatively, the baselines in the ECEF reference frame and the summation of integer ambiguities and line biases can be determined first by using a linear least-squares fit. Then, the integer ambiguities and line biases can be separated, without loss of any information, by taking the integer parts as the integer ambiguities.

\subsection{Integer Ambiguity Resolution}

The integer ambiguities can be determined using either instantaneous or dynamic techniques [14]. Instantaneous techniques find a solution that minimizes the error residual at a specific time by searching through all possible integer sets. Refinements can be made to the solution by restricting the search space using geometric constraints [8]. This is well suited to short baselines, however, the minimum residual does not guarantee a correct solution in the presence of measurement noise $[5,6]$. Hence, an instantaneous algorithm can report wrong integers as valid ones. This may cause significant problems during the self survey. Dynamic techniques perform a batch estimation using collected data for a given period of time while the integer ambiguities remain constant over the collection period. Since these techniques require that a certain amount of motion has occurred, several minutes of collection time may be required for convergence. Also, large matrix inversions may need to be taken, which may lead to numerical errors. However, dynamic techniques are more robust than instantaneous techniques because dynamic techniques have numerous checks that can be implemented into the solution before it is accepted [14].

Cohen [14] developed an algorithm that uses a linearized iterative batch estimator. By varying the sample rate and the data collection period, this algorithm can be applied for almost any vehicle motion. However, there are several disadvantages, including: 1) an a priori attitude must be given, 2) for large initial attitude errors it may converge to wrong estimates, and 3) depending on the amount of data large-order matrix inversions may be required. In Ref. [6] an algorithm has been developed that has advantages over Cohen's method, including: 1) it doesn`t require any a priori attitude information, 2) large matrix inversions are not required, and 3) it is non-iterative. Also, a covariance expression has been derived that can be used to check the integrity of the determined integer ambiguity. However, this algorithm assumes that at least three non-coplanar baselines exist. Also, a significant amount of vehicle motion is still required in order for the integers to be observable. In Ref. [7] 
a real-time attitude-independent ambiguity resolution algorithm has been developed, based on an Unscented filter formulation. This approach does allow for three coplanar baselines to exist, while providing accurate estimates that are robust to initial guess errors.

In the self survey, the antenna set connected to the receiver is not moving generally. Therefore, motion based dynamic techniques are not efficient since the sightlines are moving slowly. Instead, a fast integer ambiguity resolution algorithm shown in Ref. [8] can be used. This algorithm uses a geometric inequality to reduce the integer search space, which can be applied for coplanar baselines as well. Then, a batch-type loss function is used to resolve the integer ambiguities with a covariance integrity check. Therefore, even with a few data points the integer ambiguities can be resolved successfully. Instantaneous algorithms have an advantage in that they provide integers directly at a specific time, although they are prone to noise errors, which can induce either incorrect solutions or no solution. An integer search is performed to maximize the probability that a unique solution is the correct solution [5], while at the same time reducing the search space by using normality constraints as well as geometric constraints. Also, an attitude-independent algorithm using an effective measurement model is developed in Ref. [8] to directly determine the integers without precomputing the sightline vector in the body frame or baselines in the reference frame. The integers for all sightlines and baselines can thus be determined instantaneously or using a small batch of data.

\section{New Self Survey Approach}

Previous self survey approaches mostly rely on determining the survey parameters in one batch algorithm. This approach has several disadvantages. Mainly, good estimates must usually be known a priori. The new approach uses an attitude-independent method to first resolve the integers, thereby making it more robust. Also, some algorithms do not allow for changes in baseline length. Changes in the baseline lengths due to the phase differences from the geometric centers may result in large estimation errors. Furthermore, the previous integer ambiguity resolution algorithms do not work if line bias errors are present in the measurements. In the new approach, these problems are solved using a double difference technique with the Levenberg-Marquardt method. Also, cycle slip detection and a repair subsystem are added. A flowchart of the new approach is shown in Figure 2, where $M$ is the total number of sightlines and $z_{i j}$ will be defined later. 


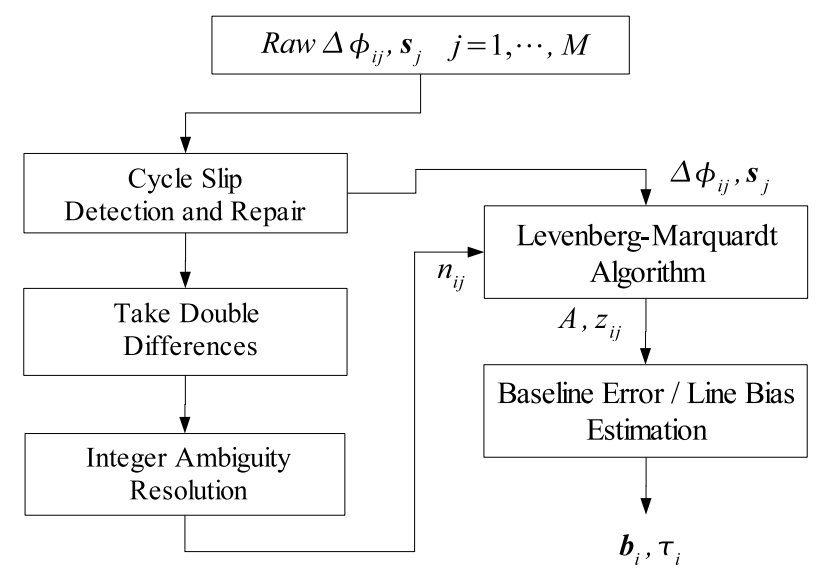

Figure 2: Flowchart of the New Self Survey Algorithm

\subsection{Cycle Slip Detection and Repair}

When a receiver acquires a GPS signal for the first time, the fractional part of the phase difference between the satellite transmitted carrier and a receiver generated replica signal is observed, and an integer counter is initialized [1]. During the tracking, the counter is incremented by one cycle whenever the fractional phase changes from 1 to 0 . The initial integer number, $n$, of cycles between the satellite and the receiver remains constant as long as no loss of signal lock occurs. When the signal lock is lost, the integer counter is restarted. Therefore, a cycle jump, called cycle slip, may occur. Sources of cycle slips are: 1) obstruction of GPS signal due to trees, buildings, mountains, etc., 2) a low signal-to-noise ratio due to weak signals, high multipath, or signal tracking errors, or 3) a failure in the receiver software or hardware [1].

Single differences of the phase measurements collected by a Trimble Advanced Navigation System (TANS) Vector receiver and their cycle slip repaired counterparts are shown in Figure 3. As can be seen from this figure, initial and end raw measurements contain numerous cycle slips with signal lock losses. Also, the duration of signal lock loss lasts several minutes for this case. Therefore, the determination of cycle slip size becomes complicated. However, by monitoring its time derivative, large signal lock loss cases can be successfully compensated.

An example of the time derivative comparison between the measurements and the estimates is shown in Figure 4. The estimates are determined by fitting a simple polynomial to the measurements using a least squares algorithm. A first-order polynomial fit works successfully for the early 30 to 50 minutes of data because the sightlines are moving slowly for the static receiver case. After then, a real-time sequential estimator is used for the cycle 


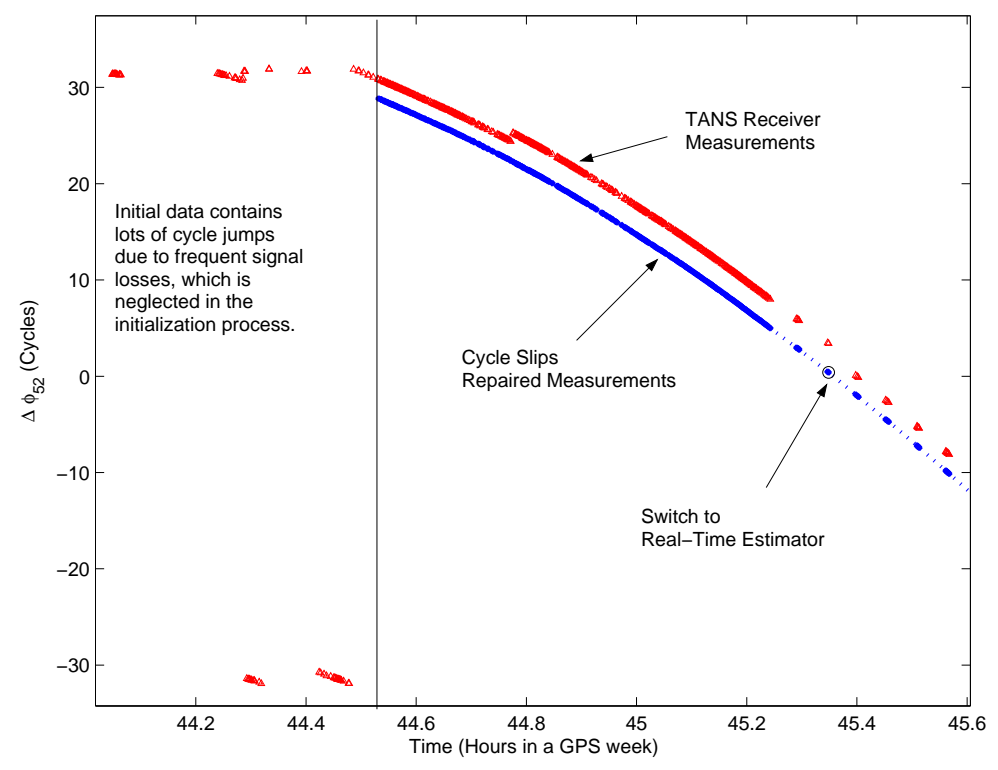

Figure 3: Measured and Cycle Slips Repaired $\Delta \phi$ Example

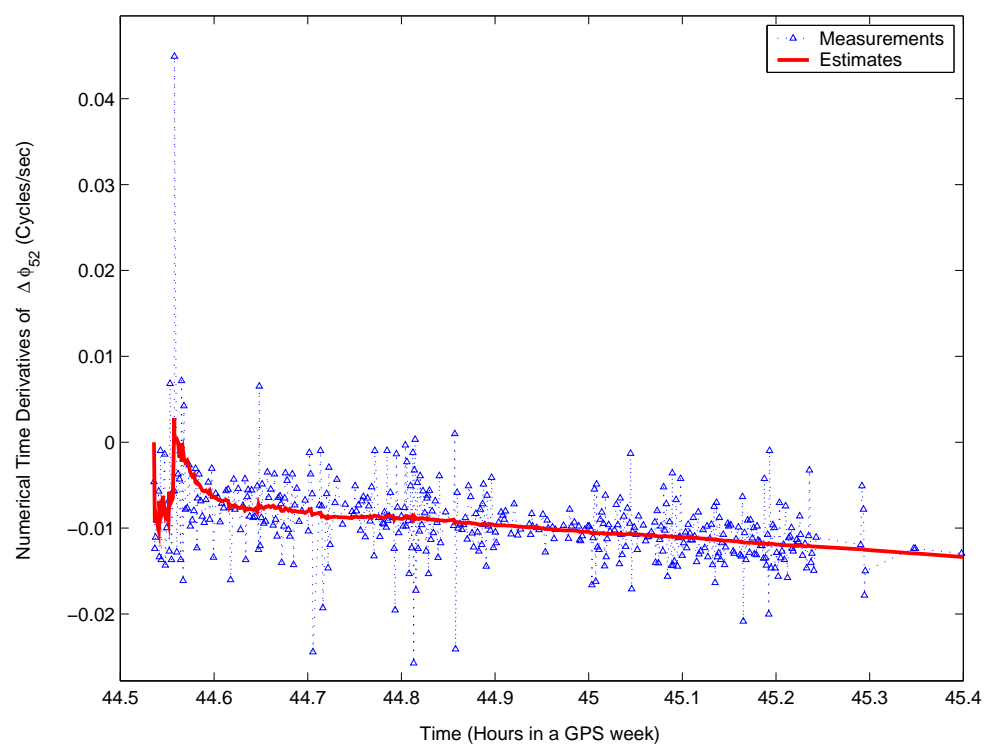

Figure 4: Measured and Estimated $\Delta \dot{\phi}$ Example

slip detection and repair, since the slope of the time derivative changes. Since a higher-order polynomial fit is required, at least 30 minutes of data are needed for the initialization of the estimator. In real data applications an 8-th order polynomial is sometimes required for a correct result. However, this order does not pose any problems in the sequential estimator solution. 


\subsection{Integer Ambiguity Resolution}

The integer ambiguities need to be resolved during the self survey. However, existing integer ambiguity algorithms cannot be applied due to the line bias errors present in the measurements. This problem can be resolved by taking double differences, because the line bias errors cancel in the double differenced phase measurements [15]. Still, the double differenced integer ambiguities still need to be resolved. A modified fast integer ambiguity resolution algorithm is derived here for this purpose. Since double differences between sightlines are applied, the cost function and the geometric constraint of Ref. [8] are reconstructed. After resolving the double differenced integer ambiguities, the baselines in the reference frame and line biases can be determined using least squares. Finally, the integer ambiguities of the single differenced phase measurements can be obtained by taking the integer part of the residual between the measurement and the dot product of the baseline estimates and the sightlines.

\subsubsection{Double Differences}

For single differenced carrier phase measurements, line biases in the baselines are troublesome. This problem can be resolved by taking between-receiver, between-sightlines double difference measurements. If the differences of the single differences in the sightlines $j$ and $k$ can be taken, the line bias on the $i$-th baseline, $\tau_{i}$ would be eliminated. Therefore, the double differenced phase measurements can be written by

$$
{ }^{2} \Delta \tilde{\phi}_{i}^{j k}=\mathbf{b}_{i}^{\top} A\left(\boldsymbol{s}_{j}-\boldsymbol{s}_{k}\right)-\left(n_{i j}-n_{i k}\right)+\left(\epsilon_{i j}-\epsilon_{i k}\right)
$$

where ${ }^{2} \Delta \tilde{\phi}_{i}^{j k}$ denotes the double differenced phase between single differenced phases $\Delta \tilde{\phi}_{i j}$

and $\Delta \tilde{\phi}_{i k}$, and $\epsilon_{i j}$ denotes the phase measurement error. Note that the measurement noise increases by a factor of $\sqrt{2}$ times that of the single difference noise though. Still, double differenced phase measurements are useful since line biases do not need to be determined.

\subsubsection{Geometric Constraint for Double Differences}

Using double differenced phase measurements, the identity of the three-dimensional vectors in Ref. [8] pertaining to the geometric constraint for single differenced phase measurements 
is rewritten as

$$
\begin{aligned}
{\left[\left(A \boldsymbol{s}_{j k}\right) \cdot\left(\mathbf{b}_{1} \times \mathbf{b}_{2}\right)\right]^{2}=} & \left(A \boldsymbol{s}_{j k} \times \mathbf{b}_{1}\right) \cdot\left[\left(\mathbf{b}_{1} \times \mathbf{b}_{2}\right) \cdot\left(\mathbf{b}_{2} \times A \boldsymbol{s}_{j k}\right)\right] \\
= & \left(A \boldsymbol{s}_{j k}\right)^{2}\left(\mathbf{b}_{1}\right)^{2}\left(\mathbf{b}_{2}\right)^{2}-\left(A \boldsymbol{s}_{j k}\right)^{2}\left(\mathbf{b}_{1} \cdot \mathbf{b}_{2}\right)^{2} \\
& -\left(\mathbf{b}_{1}\right)^{2}\left(A \boldsymbol{s}_{j k} \cdot \mathbf{b}_{2}\right)^{2}-\left(\mathbf{b}_{2}\right)^{2}\left(A \boldsymbol{s}_{j k} \cdot \mathbf{b}_{1}\right)^{2} \\
& +2\left(A \boldsymbol{s}_{j k} \cdot \mathbf{b}_{2}\right)\left(A \boldsymbol{s}_{j k} \cdot \mathbf{b}_{2}\right)\left(\mathbf{b}_{1} \cdot \mathbf{b}_{2}\right) \\
= & \left\|\boldsymbol{s}_{j k}\right\|^{2}\left\|\mathbf{b}_{1}\right\|^{2}\left\|\mathbf{b}_{2}\right\|^{2}-\left\|\boldsymbol{s}_{j k}\right\|^{2}\left(\mathbf{b}_{1} \cdot \mathbf{b}_{2}\right)^{2} \\
& -\left\|\mathbf{b}_{1}\right\|^{2}\left({ }^{2} \Delta \tilde{\phi}_{2}^{j k}+n_{2}^{j k}\right)^{2}-\left\|\mathbf{b}_{2}\right\|^{2}\left({ }^{2} \Delta \tilde{\phi}_{1}^{j k}+n_{1}^{j k}\right)^{2} \\
& +2\left({ }^{2} \Delta \tilde{\phi}_{1}^{j k}+n_{1}^{j k}\right)\left({ }^{2} \Delta \tilde{\phi}_{2}^{j k}+n_{2}^{j k}\right)\left(\mathbf{b}_{1} \cdot \mathbf{b}_{2}\right) \\
> & 0
\end{aligned}
$$

where $\boldsymbol{s}_{j k}=\boldsymbol{s}_{j}-\boldsymbol{s}_{k}$ and $n_{i}^{j k}$ is the double differenced integer for the $i$-th baseline between the $j$-th and $k$-th sightlines. Although double differences may increase the search space size twice as much as using single differences, a significant reduction of the search space size is achieved by using the constraint in Eq. (3). For example, with three baselines (assuming that $\kappa$ denotes all possible integers associated with each baseline) the search space required to determine the integers is on the order of $(2 \kappa)^{3}$; however, with the reduced subset using Eq. (3) the search space is now on the order of $3(2 \kappa)^{2}$ [5]. Furthermore, by taking the common integers corresponding to each baseline, found between any two baseline pairs, the search space becomes much smaller. In a few meters baselines scenario, it was found that the number of integers that pass the geometric constraint test is approximately $5 \% \sim 40 \%$ of the total number.

\subsubsection{Integer Ambiguity Resolution}

After reducing the search space, the double differenced integer ambiguities in Eq. (3) can now be resolved using the cost function in Eq. (5), which is modified from the cost function in Ref. [8] for double differenced phase measurements. Since three baselines are used, the double differenced integer ambiguities contained in the double differenced phase between the $j$-th sightline and the $k$-th sightline can be defined by

$$
\mathbf{n}^{j k} \equiv\left[n_{1}^{j k}, n_{2}^{j k}, n_{3}^{j k}\right]^{\top}
$$


Then, the modified cost function is given by

$$
\begin{aligned}
& J\left(\mathbf{n}^{j k}\right)=\frac{1}{2} \sum_{m=1}^{L}\left\{\frac{1}{\sigma_{j k}^{2}(m)}[\right.\left\|S_{j k}^{-1}(m) \Gamma_{j k}(m)\left({ }^{2} \Delta \tilde{\mathbf{\Phi}}^{j k}(m)-\mathbf{n}^{j k}\right)\right\|^{2} \\
&\left.\left.-\left\|\mathbf{b}_{i}\right\|^{2}+\operatorname{trace}\left\{S_{j k}^{-1}(m)\right\}\right]^{2}+\log \sigma_{j k}^{2}(m)\right\}
\end{aligned}
$$

with

$$
\begin{gathered}
\sigma_{j k}^{2}=\left({ }^{2} \Delta \tilde{\mathbf{\Phi}}^{j k}-\mathbf{n}^{j k}\right)^{\top} \Gamma_{j k}^{\top} S_{j k}^{-3} \Gamma_{j k}\left({ }^{2} \Delta \tilde{\mathbf{\Phi}}^{j k}-\mathbf{n}^{j k}\right)-\operatorname{trace}^{2}\left\{S_{j k}^{-1}\right\} \\
\Gamma_{j k} \equiv\left[\varpi_{12}^{-2} \boldsymbol{s}_{12}, \varpi_{13}^{-2} \boldsymbol{s}_{13}, \varpi_{14}^{-2} \boldsymbol{s}_{14}\right] \\
{ }^{2} \Delta \tilde{\boldsymbol{\Phi}}^{j k} \equiv\left[{ }^{2} \Delta \tilde{\phi}_{1}^{j k},{ }^{2} \Delta \tilde{\phi}_{2}^{j k},{ }^{2} \Delta \tilde{\phi}_{3}^{j k}\right]^{\top} \\
S_{j k}=\varpi_{12}^{-2} \boldsymbol{s}_{12} \boldsymbol{s}_{12}^{\top}+\varpi_{13}^{-2} \boldsymbol{s}_{13} \boldsymbol{s}_{13}^{\top}+\varpi_{14}^{-2} \boldsymbol{s}_{14} \boldsymbol{s}_{14}^{\top}
\end{gathered}
$$

where $\varpi_{j k}$ is the weighting for the double differenced phase measurements ${ }^{2} \Delta \tilde{\boldsymbol{\Phi}}^{j k}$, which is given by $\sqrt{2}$ times the standard deviation of the measurement error for single differences, i.e., $\varpi_{j k}=0.026 \sqrt{2}$ cycles. Also, $L$ is the total number of data points (when $L=1$ an instantaneous solution is provided). As can be seen in Eq. (6b), only three double differenced sightlines are used because three baselines need to be determined. Equation (5) can be used to determine the double differenced integer ambiguities instantaneously or using a small amount of data (i.e. $L>1$ ), by checking the remaining integers that pass the inequality condition in Eq. (3). The integer set that minimizes Eq. (5) is chosen as the final solution. Then, for further applications the integer ambiguities, $n_{i j}$, contained in the single differenced phase measurements need to be resolved. First, the baselines in the reference frame are estimated using linear least squares that minimize the cost function given by

$$
J\left(\overline{\mathbf{b}}_{i}\right)=\frac{1}{2} \sum_{\text {all } j k} \varpi_{j k}^{-2}\left({ }^{2} \Delta \tilde{\phi}_{i}^{j k}-\overline{\mathbf{b}}_{i}^{\top} \boldsymbol{s}_{j k}\right)^{2}
$$

where $\overline{\mathbf{b}}_{i}$ is the $i$-th baseline in the reference frame. The solution to minimize Eq. (7) is straightforward [3], which is given by

$$
\overline{\mathbf{b}}_{i}=N_{i}^{-1} \boldsymbol{y}_{i}
$$

where

$$
N_{i}=\sum_{\text {all jk }} \varpi_{j k}^{-2} \boldsymbol{s}_{j k} \boldsymbol{s}_{j k}^{\top}
$$




$$
\boldsymbol{y}_{i}=\sum_{\text {all } j k} \varpi_{j k}^{-2}{ }^{2} \Delta \tilde{\phi}_{i}^{j k} \boldsymbol{s}_{j k}
$$

After determining the baselines in the reference frame, the integer ambiguities of the single differential phase measurements are resolved by taking the integer part of the residual given by

$$
n_{i j}=\text { floor }\left(\Delta \tilde{\phi}_{i j}-\overline{\mathbf{b}}_{i}^{\top} \boldsymbol{s}_{j}\right)
$$

where floor is the MATLAB command which rounds the residual to the nearest integer towards minus infinity. This approach may not resolve the integers if the line bias errors are close to 0 or 1 , however, for this case the line bias errors can be considered as integer ambiguities.

\subsection{Levenberg-Marquardt Algorthm}

Since the integer ambiguities now are resolved, Eq. (1) can be rewritten as

$$
\Delta \phi_{i j}{ }^{\prime}={ }^{g} \mathbf{b}_{i}^{\top} A \boldsymbol{s}_{j}+z_{i j}
$$

where ${ }^{g} \mathbf{b}$ represents the geometric baseline vector in the body frame that connects the geometric center of two antennas, $\Delta \phi_{i j}{ }^{\prime}=\Delta \phi_{i j}-n_{i j}$, and $z_{i j}$ is a dummy parameter used in the baseline estimation, given by

$$
z_{i j}=\Delta \mathbf{b}_{i}^{\top} A \boldsymbol{s}_{j}+\tau_{i}
$$

where $\Delta \mathbf{b}_{i} \in \mathrm{R}^{3}$ is the additive error of a baseline in the body frame. To estimate the attitude parameters and $z_{i j}$ in Eq. (11), NLS can be used. However, NLS may not converge to the correct solution unless the initial guess is close to the true value. The method of steepest descent may help to avoid this problem, however, the convergence is very poor close to the solution [16]. These difficulties can be overcome by the Levenberg-Marquardt (LM) method $[17,18]$. In the LM method, the search direction is intermediate between the steepest descent and the differential correction direction. As the scale factor goes to zero, it is equivalent to the differential correction method, however, as the scale factor goes to infinity it becomes a steepest descent search along the negative gradient of the loss function (see Ref. [17] for more details).

By using the Modified Rodriguez Parameters (MRPs) [19] as attitude parameters, the 
optimal estimates are obtained to minimize the loss function given by

$$
J(\hat{\mathbf{p}}, \boldsymbol{z})=\sum_{i=1}^{N} \sum_{j=1}^{M}\left[\Delta \widetilde{\phi}_{i j}^{\prime}-\Delta \phi_{i j}{ }^{\prime}(\hat{\mathbf{p}}, \boldsymbol{z})\right]^{2}
$$

where $\hat{\mathbf{p}} \in \mathrm{R}^{3}$ denotes MRP estimates and $\boldsymbol{z}$ represents a row vector of which an element is $z_{i j}$. The Jacobian matrix, $H$, used in the NLS and LM algorithms is given by [19]

$$
H=\left[\begin{array}{c}
4^{g} \mathbf{b}_{i}^{\top}\left[A(\mathbf{p}) \boldsymbol{s}_{j} \times\right] \\
\underbrace{\text { total } M \text { sightlines }}_{(j-1) \text { zeros before } 1} \\
\vdots
\end{array}\right] \text { for } i=1,2,3
$$

where

$$
[\mathbf{a} \times] \equiv\left[\begin{array}{rrr}
0 & -a_{3} & a_{2} \\
a_{3} & 0 & -a_{1} \\
-a_{2} & a_{1} & 0
\end{array}\right]
$$

\subsection{Baseline Estimation}

By using the estimation results of the LM method, the differences in the baselines in the body reference frame and the line bias errors are determined. Referring to Eq. (12), linear least squares is sufficient to estimate the baseline differences and line bias errors since $\boldsymbol{z}$ can be written by

$$
\boldsymbol{z}=\left[\begin{array}{c}
\boldsymbol{s}_{1}^{\top} A^{\top}, 1 \\
\vdots \\
\boldsymbol{s}_{M}^{\top} A^{\top}, 1
\end{array}\right]\left[\begin{array}{c}
\Delta \mathbf{b}_{i} \\
\tau_{i}
\end{array}\right]
$$

Then, the baselines in the body frame are determined by

$$
\mathbf{b}_{i}={ }^{g} \mathbf{b}_{i}+\Delta \mathbf{b}_{i}
$$

Since the antenna phase errors can be as much as $2 \mathrm{~cm}$, baseline estimation is important in the self survey if the baselines are short [20]. 


\section{Simulation Results}

To test the new algorithm, Monte-Carlo like simulations are used. Eight hours of phase measurement data are generated using the '351.al3' SEM GPS almanac data, three baselines shown in section 5 and the phase measurement model shown in Figure 1. Then, 100 different random attitude matrices and line biases are generated to simulate the phase measurements. Multipath errors are not considered in the measurement data. Also, 100 different large initial guesses errors are applied.

The integers are resolved first using the double differences technique, since the cycle slips are not considered in the simulated measurements. Then, both the NLS and LM algorithms are applied for the nonlinear estimation to compare the convergence behavior. From the simulation results, comparing the estimation errors, there are no significant differences between the solutions from the NLS and LM algorithms for small initial errors. The NLS algorithm converges faster than LM algorithm; however, the convergence to the correct estimate is not guaranteed for the NLS algorithm, as shown by the simulation results. For the comparisons using large initial attitude errors, the NLS algorithm fails two times out of 100 simulations while the LM algorithm works successfully every time. A comparison of the number of iterations required for each algorithm is shown in Figure 5.

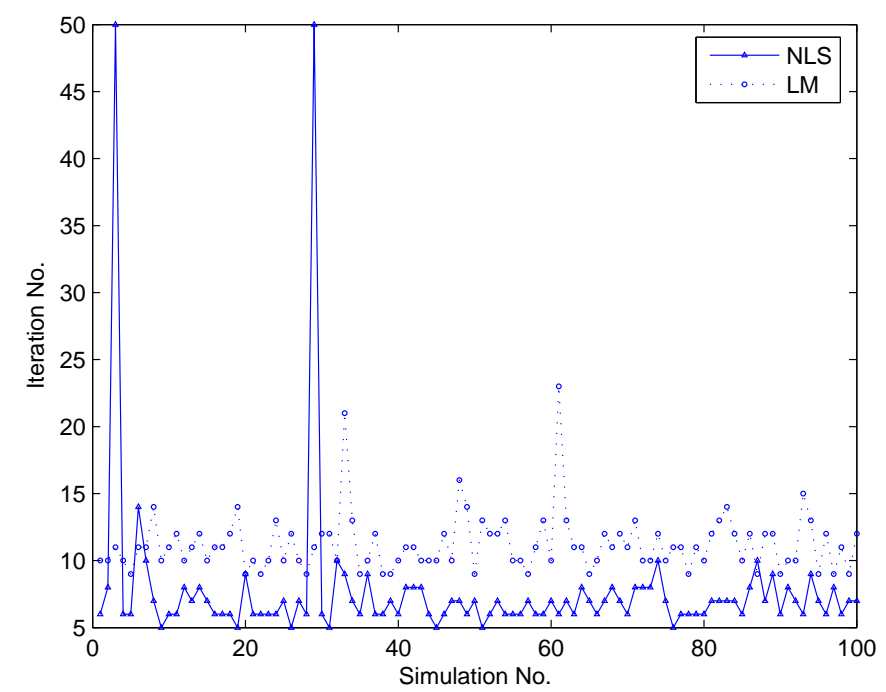

Figure 5: Iteration Number Comparison Between NLS and LM

In the following, the self survey errors between the NLS and LM algorithms are shown. Figure 6 shows the Euler angles errors, derived from the MRP errors, and their associated 
3- $\sigma$ bounds derived from the covariance matrix. The triangle marker represents the NLS Euler angle errors and the circle marker denotes the LM Euler angle errors. As can be seen from Figure 6, both the NLS and LM algorithms show the same level of estimation errors. For the pitch axis, the errors are well inside the 3- $\sigma$ boundary bounds, however, for other axes some errors are outside the 3- $\sigma$ bounds. This is because the baselines are nearly coplanar, which are aligned with body $x$-y axes. Also, the geometry of the sightlines affects the covariance. Figure 7 shows the line bias errors obtained by both the NLS and
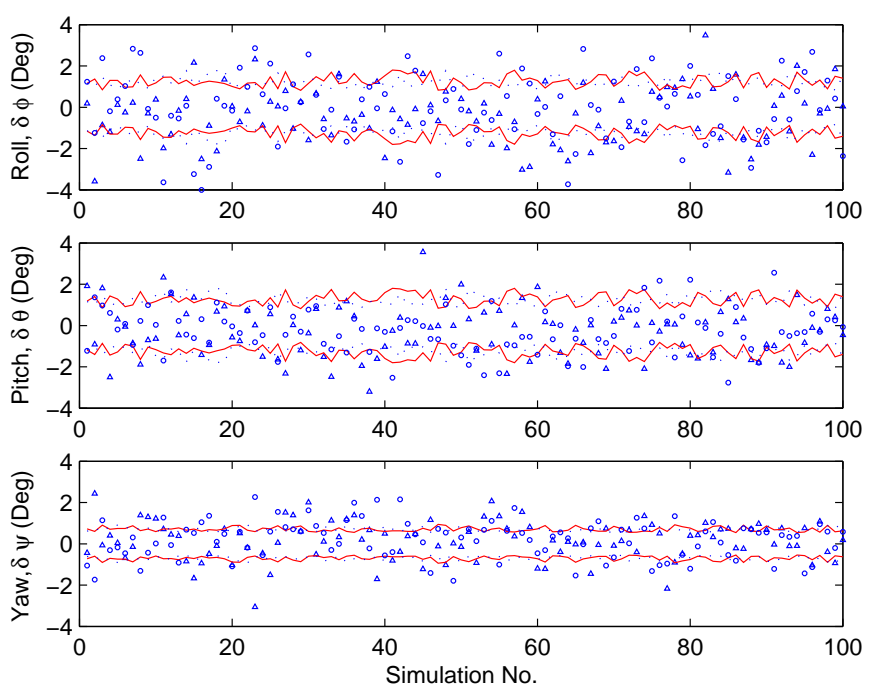

Figure 6: Attitude Error Comparison Between NLS and LM

LM algorithms. The line bias errors are well below the $3-\sigma$ bounds for baselines 2 and 3 . The line bias in the baseline 1 is not; however, it is still below the $3-\sigma$ bound of the phase measurement errors of $3 \times 0.026$. Also, the third baseline estimation errors are shown in Figure 8. As can be seen from this figure, all estimation errors are well inside their respective $3-\sigma$ bounds.

In the simulation study, the convergence performance of the LM algorithm for relatively large initial errors seems to be improved over the NLS algorithm. To compare the convergence behaviors of the LM and NLS algorithms, large initial errors are considered as

$$
\left[\begin{array}{c}
\delta \phi \\
\delta \theta \\
\delta \psi
\end{array}\right]=\left[\begin{array}{r}
-106.07^{\circ} \\
73.247^{\circ} \\
153.15^{\circ}
\end{array}\right],\left[\begin{array}{l}
\Delta \tau_{1} \\
\Delta \tau_{2} \\
\Delta \tau_{3}
\end{array}\right]=\left[\begin{array}{r}
.0547 \\
0.9129 \\
0.5019
\end{array}\right] \text { (Cycles) }
$$



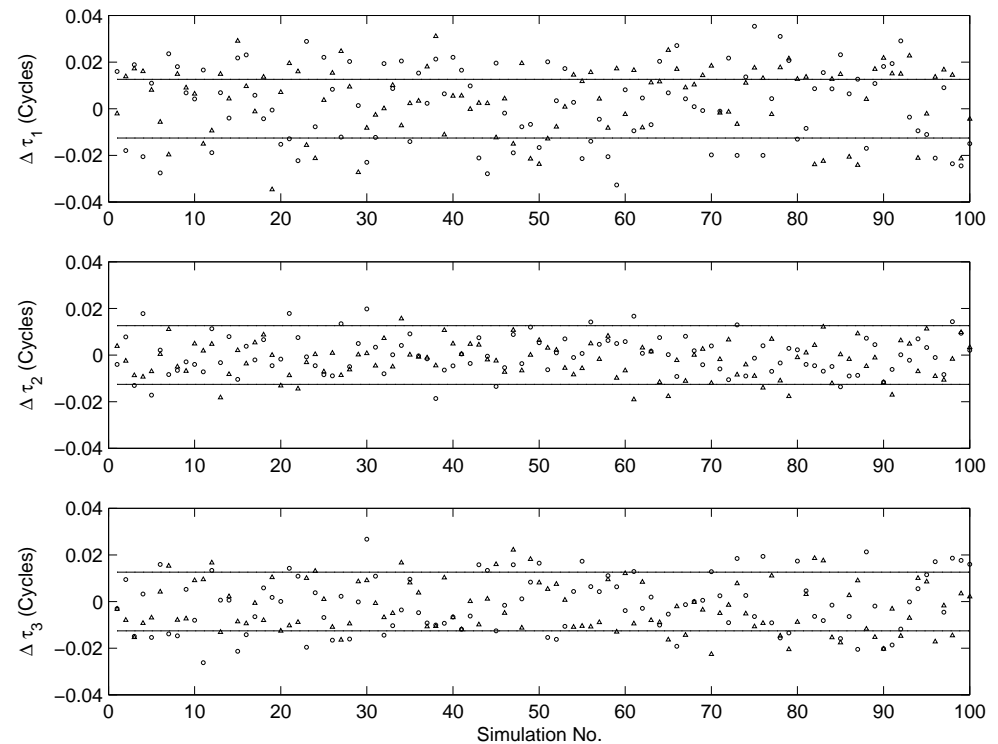

Figure 7: Line Bias Error Comparison Between NLS and LM
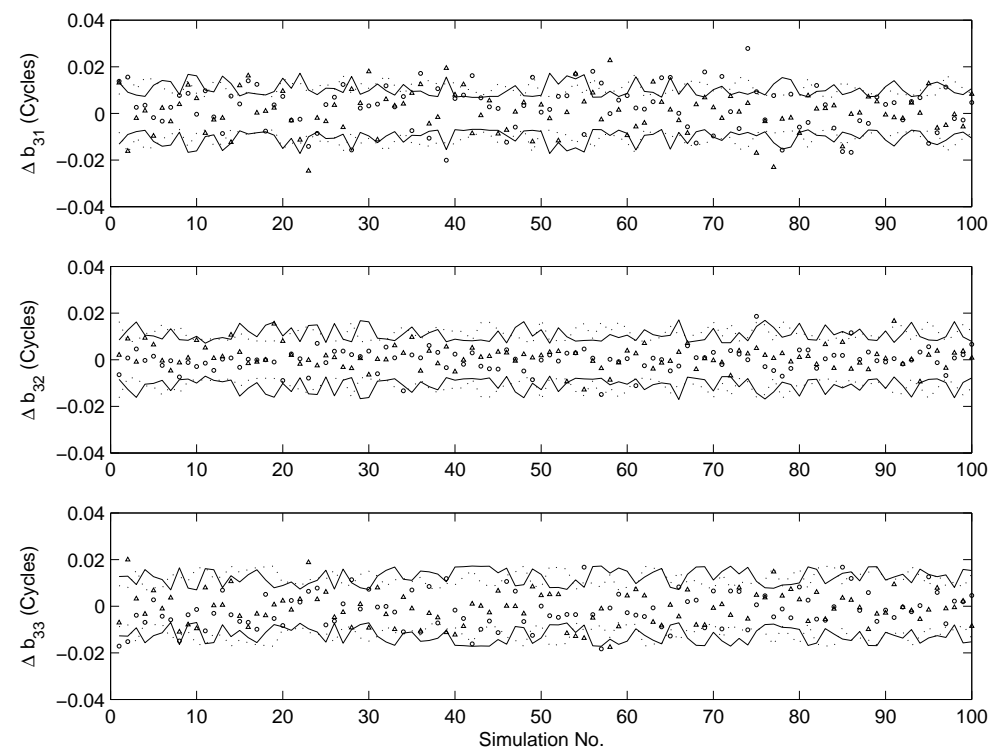

Figure 8: Baseline Error Comparison Between NLS and LM

where $\delta \phi, \delta \theta$ and $\delta \psi$ are Euler angle errors, which are converted to MRP errors. The convergence properties of the NLS and LM algorithms are compared in Figure 9. The cost function, $J$, values of each iteration for both the NLS and LM algorithms are shown. As can be seen from this figure, the iteration number required for the LM algorithm to converge is larger than that of NLS; however, the LM algorithm converges to the correct estimates after 16 iterations, while the NLS algorithm does not converge to the correct estimates. 


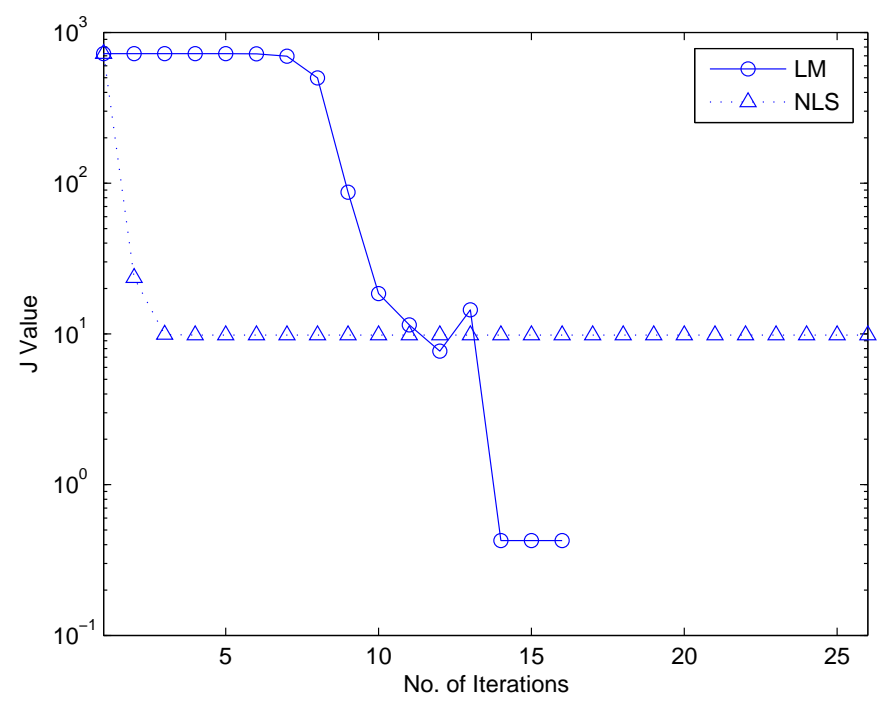

Figure 9: Convergence Comparison Between NLS and LM

\section{Real Data Application}

The new self survey algorithm is applied with real data collected by the Navigation Systems and Technology Laboratory (NSTL) at NASA's Johnson Space Center (JSC) in Houston, Texas. A TANS Vector receiver with a four antenna set is used for the test. To minimize the adverse effects from multipath errors, the antennas are installed on the roof of a building. Since the TANS Vector receiver provides an internal self survey solution, the result of new algorithm is compared with it. It should be noted that the self survey algorithm used by the TANS Vector is unknown. It is merely used to compare results with the new algorithm, which has been shown to be robust to large initial guess errors in the previous simulations.

The baselines in the East-North-Up (ENU) coordinate system shown in Figure 10 are given by

$$
\left[{ }^{g} \mathbf{b}_{1}{ }^{g} \mathbf{b}_{2}{ }^{g} \mathbf{b}_{3}\right]=\left[\begin{array}{rrr}
-115.91775 & -42.66246 & -20.74898 \\
-45.57186 & 114.46932 & -135.93129 \\
-11.68865 & -7.79448 & -12.01909
\end{array}\right] \quad(\text { Cycles })
$$

Although the baselines between geometric centers are not known, the TANS Vector receiver baselines output can be used as ${ }^{g} \mathbf{b}$. The phase measurements from the TANS Vector receiver have a range of $-32 \sim 32$ cycles. Since the lengths of the baselines are longer than 32 cycles, the phase measurement jumps to -32 cycles when it reaches 32 cycles or vice versa. Thus, 


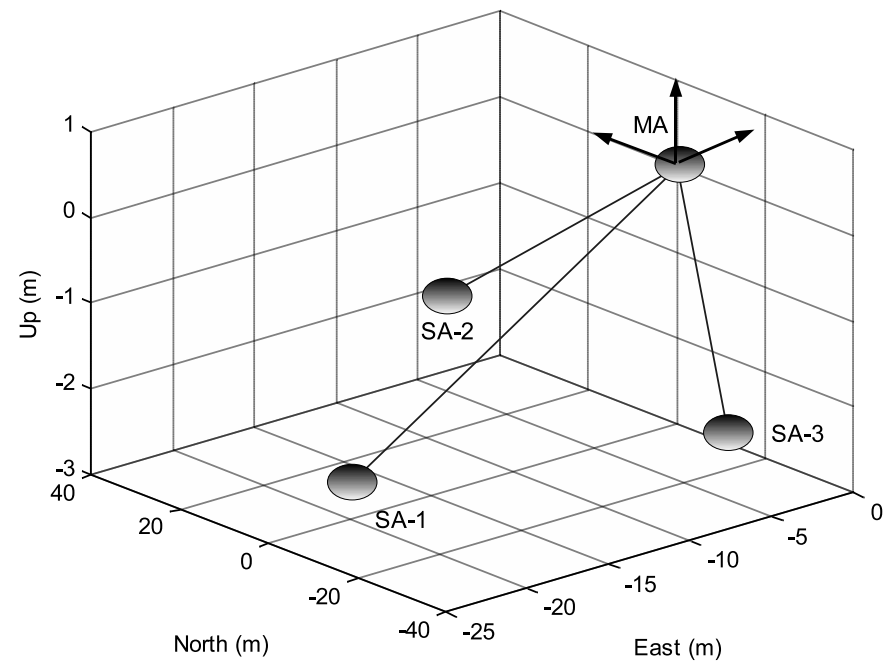

Figure 10: Baselines in the ENU Coordinate System

jumps of 64 cycles need to be compensated. Also, cycle slips are detected and repaired by the algorithm described in subsection 3.1. Nonlinear least squares converges after 9 iterations. The values of the change in the loss function, denoted by $\Delta J$, after each iteration is shown in Table 1. The resolved integer ambiguities are shown in Table 2. The differences in the

Table 1: Convergence of Nonlinear Least Squares

\begin{tabular}{|c|c|c|}
\hline Iteration & $J$ & $\Delta J$ \\
\hline \hline 1 & $2.6512 \mathrm{e}+007$ & $2.6512 \mathrm{e}+007$ \\
2 & $1.0413 \mathrm{e}+007$ & $1.6098 \mathrm{e}+007$ \\
3 & $4.8126 \mathrm{e}+006$ & $5.6007 \mathrm{e}+006$ \\
4 & $8.0795 \mathrm{e}+004$ & $4.7318 \mathrm{e}+006$ \\
5 & $9.0319 \mathrm{e}+003$ & $7.1763 \mathrm{e}+004$ \\
6 & $1.4622 \mathrm{e}+002$ & $8.8857 \mathrm{e}+003$ \\
7 & $6.9967 \mathrm{e}-001$ & $1.4552 \mathrm{e}+002$ \\
8 & $6.9942 \mathrm{e}-001$ & $2.5235 \mathrm{e}-004$ \\
9 & $6.9942 \mathrm{e}-001$ & $7.2299 \mathrm{e}-011$ \\
\hline
\end{tabular}

baseline estimates using the TANS Vector and new algorithm solutions are given by

$$
\left[\Delta \mathbf{b}_{1}, \Delta \mathbf{b}_{2}, \Delta \mathbf{b}_{3}\right]=\left[\begin{array}{rrr}
0.0147 & -0.0059 & -0.0029 \\
-0.0005 & 0.0042 & 0.0098 \\
-0.0171 & -0.0125 & -0.0188
\end{array}\right] \quad(\text { Cycles })
$$

The differences are well below the $3-\sigma$ bound of the phase measurement errors. The line bias estimates are compared with the TANS Vector receiver output in Table 3. The differences are less than 0.05 cycles. 
Table 2: Integer Ambiguities

\begin{tabular}{|r|r|r|r|r|r|r|r|}
\hline & $\boldsymbol{s}_{2}$ & $\boldsymbol{s}_{4}$ & $\boldsymbol{s}_{7}$ & $\boldsymbol{s}_{8}$ & $\boldsymbol{s}_{11}$ & $\boldsymbol{s}_{19}$ & $\boldsymbol{s}_{24}$ \\
\hline $\mathbf{b}_{1}$ & -73 & -46 & -114 & -24 & 58 & 56 & -81 \\
$\mathbf{b}_{2}$ & -80 & 88 & 70 & -87 & 94 & 9 & 73 \\
$\mathbf{b}_{3}$ & 46 & -147 & -118 & 80 & -63 & 33 & -116 \\
\hline
\end{tabular}

Table 3: Line Biases (Unit: Cycles)

\begin{tabular}{|c|c|c|}
\hline Line Biases & Self Survey & TANS Receiver \\
\hline$\tau_{1}$ & 0.2637 & 0.2240 \\
$\tau_{2}$ & 0.9367 & 0.8954 \\
$\tau_{3}$ & 0.3056 & 0.3196 \\
\hline
\end{tabular}

The determined attitude matrix is given by

$$
A=\left[\begin{array}{rrr}
-0.0003 & -0.6662 & 0.7457 \\
-0.2844 & 0.7150 & 0.6387 \\
-0.9587 & -0.2119 & -0.1897
\end{array}\right]
$$

By using the estimated baselines, line biases and attitude matrix, the single differenced phases are computed and compared with the measurement data. Figures 11 through 13 show the residual errors between the measured and the estimated phases. Since multipath errors still exist in the measurements, oscillations are shown in the residual error. Also, the residual errors increase both in the early part and in the end data. However, in the other regions the residual errors are below the measurement $3-\sigma$ bound of $3 \times 0.026$. Figure 11
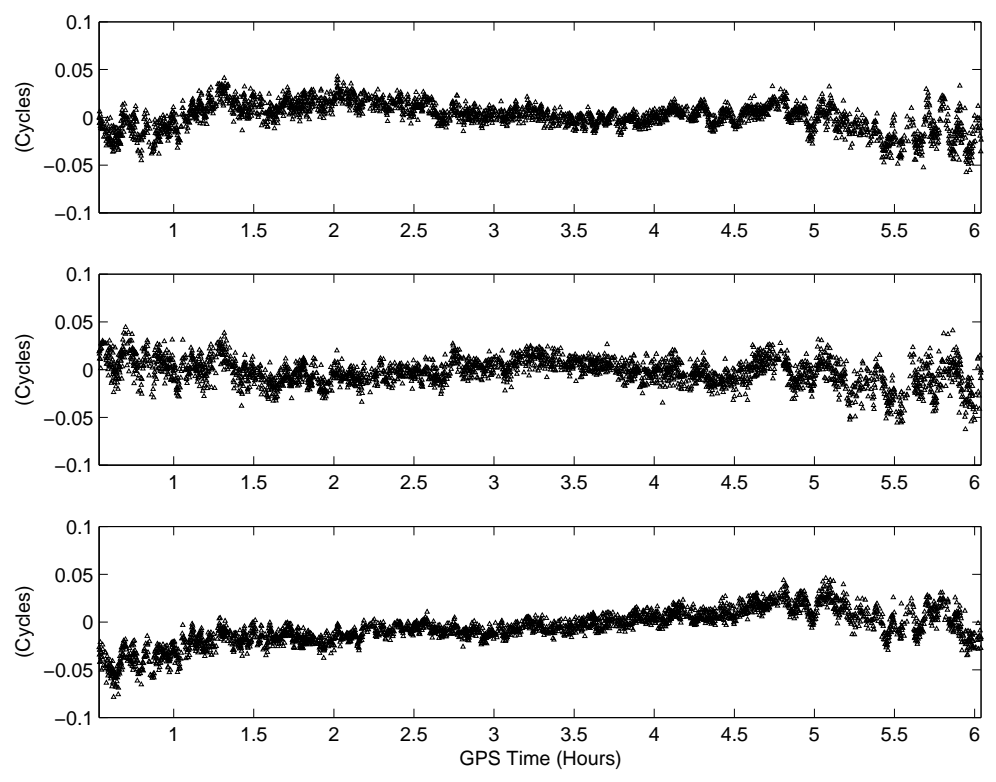

Figure 11: The Residual Error of $\widetilde{\Delta \phi_{i 2}}-\Delta \phi_{i 2}$ for $i=1,2,3$ 
shows the residual errors for the pseudo random noise (PRN) 2 signal phase. The residuals between 100 and 300 minutes are well below the 3- $\sigma$ measurement bound, while the residuals before 80 minutes and after 300 minutes begin to increase. Similar trends are shown for the PRN 4, 7, 8 and 24 signal phases. However, as can be seen in Figures 12 and 13, the signal phases are different for PRN 11 and 19. There exist oscillations in the residual since multipath errors are more likely present in these measurements. Still, the magnitudes of these residuals are near the assumed noise levels of the measurements. The results obtained using the actual data indicate that the new self survey algorithm provides good overall parameter estimates.
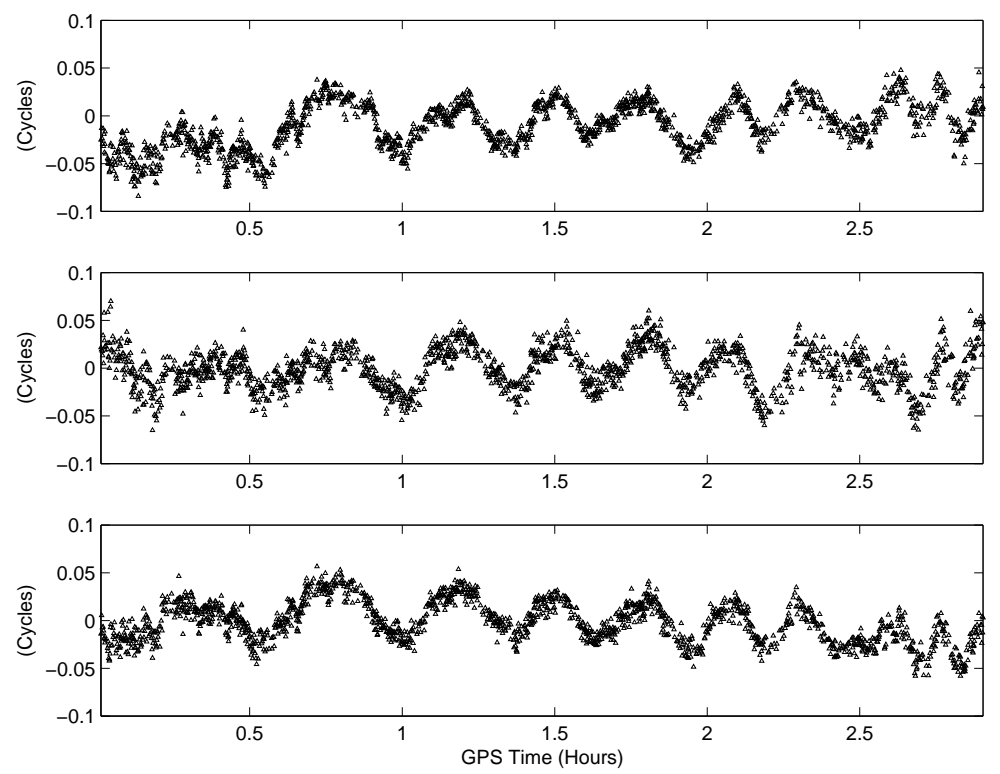

Figure 12: The Residual Error of $\widetilde{\Delta \phi_{i 11}}-\Delta \phi_{i 11}$ for $i=1,2,3$

\section{Conclusions}

A new self survey method has been developed and compared with a commercial GPS receiver self survey result. The new method includes cycle slip detection and a repair algorithm to compensate the cycle counter re-initialization problem when the GPS signal lock is lost. Also, it includes a double differencing scheme to resolve integer ambiguities when line bias errors are present in the measurements. For cycle slip detection and repair, a first-order polynomial fit is used for early-time data segments since frequent signal lock loss may occur. Then, after 30 minutes an 8-th order polynomial replaces it because the sightlines are 

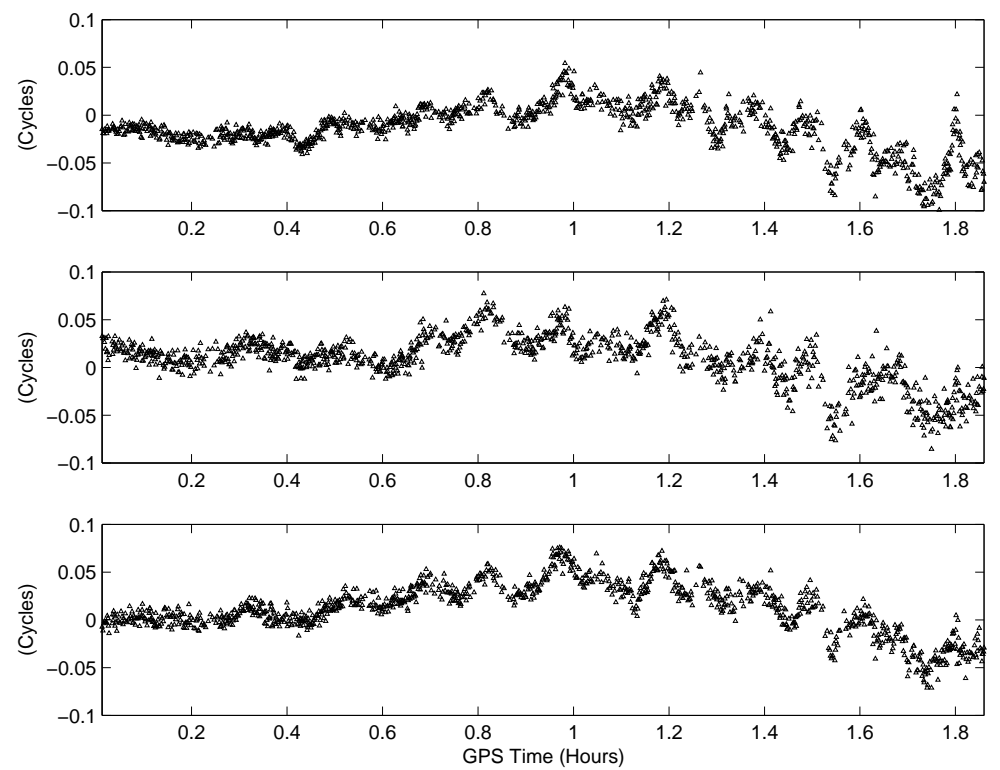

Figure 13: The Residual Error of $\widetilde{\Delta \phi_{i 19}}-\Delta \phi_{i 19}$ for $i=1,2,3$

moving. To verify the new algorithm, real data collected by the TANS Vector GPS receiver is used for the cycle slip detection and repair algorithm. Integer ambiguity resolution using double differenced phase measurements is then accomplished. Then, nonlinear least squares and the Levenberg-Marquardt algorithm are used to determine attitude parameters. Finally, baselines in the body frame and line biases are determined. In the comparison of NLS and LM algorithms using 100 simulations with random initial conditions, the LM algorithm shows more robust results for large initial errors, although the convergence speed of the NLS algorithm is faster. In the comparison with the TANS Vector receiver self survey output, the integer ambiguities match exactly. Also, line biases and baselines differences were within their respective 3- $\sigma$ error bounds.

\section{Acknowledgement}

This work was supported under a NASA grant (NASA \#5-7510), under the supervision of Ms. Janet Bell. The authors greatly appreciate this support as well as help from the Navigation Systems and Technology Laboratory at NASA - Johnson Space Center. 


\section{References}

[1] Hofmann-Wellenhof, B., Lichtenegger, H., and Collins, J., Global Positioning System: Theory and Practice, 4th ed., Springer-Verlag, New York, 1997.

[2] Cohen, C., Attitude Determination Using GPS, Ph.D. dissertation, Stanford University, Dec. 1992.

[3] Crassidis, J., Markley, F., and Lightsey, E., "Application of Vectorized Attitude Determination Using Global Positioning System Signals," Proceedings of the AIAA/AAS Astrodynamics Specialist Conference, Boston, MA, Aug. 1998.

[4] Crassidis, J., Lightsey, E., and Markley, F., "Efficient and Optimal Attitude Determination Using Recursive Global Positioning System Signal Operations," AIAA Journal of Guidance, Control, and Dynamics, Vol. 22, No. 2, March-April 1999, pp. 193-201.

[5] Park, K. and Crassidis, J., "Autonomous Attitude Determination for ISS Applications Using Pseudolite Signals," Tech. Rep., NASA Johnson Space Center, Houston, TX, June 2003.

[6] Crassidis, J., Markley, F., and Lightsey, E., "Global Positioning System Integer Ambiguity Resolution Without Attitude Knowledge," AIAA Journal of Guidance, Control, and Dynamics, Vol. 22, No. 2, March-April 1999, pp. 212-218.

[7] Lightsey, E. and Crassidis, J., "Real Time Attitude Independent GPS Integer Ambiguity Resolution," The John L. Junkins Astrodynamics Symposium, College Station, TX, May 2003, pp. 145-164.

[8] Lightsey, E., Crassidis, J., and Markley, F., "Fast Integer Ambiguity Resolution for GPS Attitude Determination," Proceedings of the AIAA Guidance, Navigation, and Control Conference, Portland, OR, Aug. 1999, pp. 403-412.

[9] Comp, C. and Axelrad, P., "Adaptive SNR-Based Carrier Phase Multipath Mitigation Technique," IEEE Transactions on Aerospace and Electric Systems, Vol. 34, No. 1, Jan. 1998, pp. 264-276.

[10] Park, K., GPS Receiver Self Survey and Attitude Determination using Pseudolite Signals, Ph.D. dissertation, Texas A\&M University, College Station, TX, Aug. 2004. 
[11] Altmayer, C., "Enhancing the Integrity of Integrated GPS/INS Systems by Cycle Slip Detection and Correction," Proceedings of the IEEE Intelligent Vehicles Symposium, Miami, FL, Oct. 2000, pp. 174-179.

[12] Crassidis, J. and Markley, F., "A New Algorithm for Attitude Determination Using Global Positioning System Signals," Journal of Guidance, Control, and Dynamics, Vol. 20, No. 5, Sept.-Oct. 1997, pp. 891-896.

[13] Bate, R. R., Mueller, D. D., and White, J. E., Fundamentals of Astrodynamics, Dover Publications, New York, NY, 1971.

[14] Cohen, C., "Attitude Determination," Global Positioning System: Theory and Applications,Vol. II, edited by B.W. Parkinson and J.J. Spilker, Jr., American Institute of Aeronautics and Astronautics, Washington, DC, 1996, pp. 519-538.

[15] Parkinson, B. and Spilker Jr., J., ed., Global Positioning System: Theory and Applications, Vol. I-II, American Institute of Aeronautics and Astronautics, Washington, DC, 1996.

[16] Bryson, Jr., A. and Ho, Y., Applied Optimal Control (Revised Printing), Taylor \& Francis, Levittown, PA, 1975.

[17] Crassidis, J. and Junkins, J., Optimal Estimation of Dynamic Systems, Chapman \& Hall/CRC, Boca Raton, FL, 2004.

[18] Altmayer, C., "Accuracy Improvements of Pseudolite Systems - First Results," Proceedings of National Technical Meeting 2001, Long Beach, CA, Jan. 2001, pp. 491-500.

[19] Shuster, M., "A Survey of Attitude Representations," Journal of Astronautical Sciences, Vol. 41, No. 4, Oct. 1993, pp. 439-517.

[20] Hayward, R. and Powell, J., "Real Time Calibration of Antenna Phase Errors for Ultra Short Baseline Attitude Systems," ION GPS-98, Nashville, TN, Sept. 1998, pp. 17531762. 